\title{
Comparison of Effect of Non-invasive Ventilation Delivered by Helmet vs Face Mask in Patients with COVID-19 Infection: A Randomized Control Study
}

\author{
Anupriya Saxena ${ }^{1}$, Nazia Nazir ${ }^{2}$, Ruchi Pandey $^{3}$, Savita Gupta $^{4} \oplus$
}

\begin{abstract}
Background and aims: We compared the effectiveness of non-invasive ventilation (NIV) provided by helmet mask vs face mask in patients with COVID-19.

Methods and materials: Between March and May 2021, a single-center, prospective, open-label randomized controlled research was undertaken. Sixty patients were randomly assigned to one of two groups based on the NIV delivery interface. In group I $(n=30)$ helmet mask was used and in group II ( $n=30)$ face mask was used for delivery of NIV. The proportion of patients in each group who required endotracheal intubation was the primary outcome. The duration of NIV, length of stay in the intensive care unit (ICU), hospital mortality, ratio of partial pressure of oxygen to fraction of inspired oxygen $\left(\mathrm{PaO}_{2} / \mathrm{FiO}_{2}\right)$, respiratory rate, patient comfort, and complications were all documented as secondary outcomes. Results: In both groups, demographics, clinical characteristics, and treatment received were comparable. Around $10 \%$ of patients in the helmet mask group were intubated, while $43.3 \%$ of patients in the face mask group were intubated $(p=0.004)$. The two groups demonstrated similar hemodynamic patterns. The use of a helmet mask, on the other hand, resulted in enhanced oxygenation ( $263.57 \pm 31.562 \mathrm{vs} 209.33 \pm 20.531$, $p=0.00)$, higher patient satisfaction $(p=0.001)$, a lower risk of complications, and a shorter NIV and ICU stay $(p=0.001)(4.53 \pm 0.776$ vs $7.60 \pm 1.354, p=0.00$ and $6.37 \pm 0.556$ vs $11.57 \pm 2.161, p=0.00)$.

Conclusion: Helmet mask could be a reliable interface for delivery of NIV in COVID-19 and results in a lower rate of endotracheal intubation, better oxygenation with greater patient comfort and shorter ICU stay as compared to face mask used for NIV.

Keywords: COVID-19, Critical care unit, Face mask, Helmet continuous positive airway pressure, Hypoxia, Non-invasive ventilation.

Indian Journal of Critical Care Medicine (2022): 10.5005/jp-journals-10071-24155
\end{abstract}

\section{INTRODUCTION}

The coronavirus disease-2019 (COVID-19) outbreak has affected millions of people all over the world. Although the majority of patients have a favorable prognosis, infection can cause acute respiratory distress syndrome (ARDS), which has a high risk of morbidity and mortality. According to oxygenation criteria, the prevalence of ARDS in COVID-19 varies from 20 to $67 \%$ in hospitalized patients and is $100 \%$ in patients who get intubated. ${ }^{1}$

Non-invasive ventilation (NIV) may play a critical role in managing patients with respiratory failure secondary to COVID-19 infection. Studies have reported that NIV has effectively prevented the need for intubation in many of the patients treated. ${ }^{2}$ Hence in COVID-19 patients, many appeared to have shifted away from early intubation and toward NIV.

The helmet mask, major and evidence-based airway equipment, was first introduced utilizing hyperbaric oxygen treatment device modifications, and then utilized for $\mathrm{NIV}^{3}$ Its use was also prompted by concerns about poor patient compliance and the risk of aerosolization from older forms of NIV. Although it is not intended to replace endotracheal intubation and mechanical ventilatory support in severely ill patients, research from Italy and China shows that COVID-19 patients managed with it may have a better outcome. ${ }^{4}$ However, these benefits have not yet been confirmed in a randomized control trial. As a result, this study was carried out to quantify the prospective benefits of a helmet mask over a face mask NIV in COVID-19 patients with ARDS.

When a helmet mask is utilized as an interface for providing NIV, we hypothesize that it can result in lesser rate of mechanical
${ }^{1}$ Anaesthesia, Government Institute of Medical Sciences, New Delhi, India

${ }^{2}$ Anesthesiology and Critical Care, Government Institute of Medical Sciences, Greater Noida, Uttar Pradesh, India

${ }^{3}$ Community Medicine, Government Institute of Medical Sciences, Greater Noida, Uttar Pradesh, India

${ }^{4}$ Department of Anaesthesia, Government Institute of Medical Sciences, Greater Noida, Uttar Pradesh, India

Corresponding Author: Anupriya Saxena, Anaesthesia, Government Institute of Medical Sciences, New Delhi, India, Phone: +91 9953090044, e-mail: anupriya.pmch@gmail.com

How to cite this article: Saxena A, Nazir N, Pandey R, Gupta S. Comparison of Effect of Non-invasive Ventilation Delivered by Helmet vs Face Mask in Patients with COVID-19 Infection: A Randomized Control Study. Indian J Crit Care Med 2022;26(3):282-287.

Source of support: Nil

Conflict of interest: None

ventilation and better oxygenation than when a face mask is used as an interface for providing NIV.

\section{Methods and Materials}

From March to May 2021, we conducted an open-label, singlecenter, randomized controlled research comprising consecutive patients hospitalized to our COVID ICU. The Helsinki Declaration of 1975 defined Ethical Principles for Medical Research with Human

o The Author(s). 2022 Open Access This article is distributed under the terms of the Creative Commons Attribution 4.0 International License (https://creativecommons. org/licenses/by-nc/4.0/), which permits unrestricted use, distribution, and non-commercial reproduction in any medium, provided you give appropriate credit to the original author(s) and the source, provide a link to the Creative Commons license, and indicate if changes were made. The Creative Commons Public Domain Dedication waiver (http://creativecommons.org/publicdomain/zero/1.0/) applies to the data made available in this article, unless otherwise stated. 
Subjects, which were followed in this clinical investigation (revised 2013).

The institutional ethics committee authorized the protocol, which was then registered on ctri.nic.in. Participants signed a written informed consent form. Patients between the age-group of 18-80 years who were conscious and alert, requiring NIV as a part of treatment of ARDS due to COVID-19 were eligible for enrolment.

Those requiring emergency intubation or cardiopulmonary resuscitation, respiratory arrest, hemodynamic instability, encephalopathy, respiratory failure due to neurologic disease or patients with any pre-existing pulmonary pathology, patients at risk of aspiration, history of claustrophobia, facial deformities, presence of tracheostomy, raised intracranial pressure, pregnancy, or those who had refused endotracheal intubation were excluded from the study.

Assuming the failure rate (proportion of patients undergoing intubation) in the Face mask group to be $61 \%$ and in the helmet mask group to be $18 \%,{ }^{5}$ the power the of study to be $80 \%, 95 \%$ confidence interval and enrolment ratio of 1:1, the sample size calculated was 19 in each group. In order to account for dropouts or refusals to participate in the study, a total of 30 participants in each group were enrolled (Sample Size Calculator by Kane SP. https:// clincalc.com/stats/samplesize.aspx ClinCalc: https://clincalc.com/ stats/samplesize.aspx).

Sixty-four patients were approached for the study, four patients denied consent so 60 patients were enrolled and randomized into two groups. A computer-generated randomization list was used for the randomization. NIV was administered to group I via a helmet mask interface $(n=30)$. NIV was administered to group II using a face mask interface $(n=30)$.

In both groups, the patients received NIV via a ventilator with a double-limbed circuit in bilevel pressure support mode (PM CARE CV 200 ventilator system).

The helmet mask (Innaccel SAANS CPAP Helmet) was a transparent hood made of polyvinyl chloride (latex free), fixed in place by straps attached to the two sides of the helmet under the arms with buckles adjusted to secure the helmet. The circumference of the patient's neck was measured, and the neck seal in the helmet mask was cut to achieve a tight but comfortable seal, resulting in a breathing circuit that was sealed off from the outside environment.

After ensuring the patient is wearing earplugs and hair cap, the expiratory limb was connected to the output port. The inspiratory limbs were connected to the input ports on either side. The plunger of the anti-asphyxiation valve was pulled till the helmet mask was inflated.

In group II, the appropriate size NIV non-vented face mask (large, medium, or small) was applied as per the facial contour of the patient. The size of the mask was measured from the level of the eye to the lower part of the lip. The dual limb circuit having separate inspiratory and expiratory limbs with a built-in exhalation port/ filter for removal of $\mathrm{CO}_{2}$ was used. After attaching the tubing to the ventilator, the mask was put over the face of the patient held with hand for some time till the patient got adjusted to the experience of NIV. The straps were then secured to the back of the head and on either side of the mask and was checked for any air leak. In both the groups, to avoid carbon dioxide rebreathing, an inspiratory fresh gas flow rate was set at more than $60 \mathrm{~L} / \mathrm{min}$ in the ventilator.

Both groups used the same NIV titration technique. First, expiratory positive airway pressure (EPAP) was steadily increased to achieve an oxygen saturation $\left(\mathrm{SpO}_{2}\right)$ of more than $92 \%$ keeping the inspired oxygen concentration of less than $60 \%$. Inspiratory positive airway pressure was adjusted gradually in a step by increase of 2-3 $\mathrm{cm} \mathrm{H}_{2} \mathrm{O}$ to reach a target respiratory rate of less than $30 / \mathrm{min}$ and tidal volume of $>6 \mathrm{~mL} / \mathrm{kg}$ without the use of accessory muscles of respiration. The ratio of NIV and high flow nasal cannula (HFNC) was set at 4:1 hour duration and was continued till the patient's condition improved or worsened.

The time of NIV was gradually lowered in patients who showed improvement in oxygenation, and the patient was weaned to either an HFNC or a non-rebreathing mask. The weaning criteria were same for both groups: patient's conscious, oriented with stable vitals, and absence of any tachypnea with respiratory rate lower than 25 breaths/min and no use of accessory muscles; arterial blood gas analysis ( $A B G$ ) showing $\mathrm{pH} \geq 7.35$; partial pressure of oxygen $\left(\mathrm{PaO}_{2}\right) \geq 80 \mathrm{mmHg}, \mathrm{SpO}_{2} \geq 94 \%$ with $\mathrm{FIO}_{2} \leq 0.40$, and EPAP of $5 \mathrm{~cm}$ water. Weaned patients were those who did not require NIV for more than 12 hours per day for 48 hours.

The patients deteriorating on NIV were considered for intubation as per the decision of the treating physician. The intubation criteria were the same for both the groups which included worsening neurological/ hemodynamic status, worsening $\mathrm{PO}_{2} / \mathrm{FiO}_{2}$ ratio, $\mathrm{SpO}_{2}<85 \%$, and respiratory rate $>35 / \mathrm{min}$ with the use of abdominal muscles, and ongoing hypoxia $\left(\mathrm{PaO}_{2}\right)<45$ $\mathrm{mmHg}$, persistent hypercapnea $\left(\mathrm{PCO}_{2}\right)>60 \mathrm{mmHg}$ (on NIV with $\mathrm{FiO}_{2} 100 \%$ ), and patient uncooperative to the face mask or helmet mask. Following intubation, ARDS protocol was followed with lung-protective ventilation.

All patients had round-the-clock monitoring of electrocardiography, heart rate, blood pressure, and oxygen saturation. Blood gases and oxygenation status $\left(\mathrm{PaO}_{2} / \mathrm{FiO}_{2}\right)$ were monitored 6 hourly in both the groups. The patients in both the groups received similar medical treatment as clinically appropriate.

Adverse events were pre specified to each group. In group I, skin ulceration at the neck seal, claustrophobia, noise disturbance, eye irritation, and helmet deflation were noted. In group II, the presence of facial ulcers was noted.

The patient's comfort was measured using a visual analog scale (VAS). ${ }^{6}$ The VAS to analyze the patient comfort is a $100 \mathrm{~mm}$ long horizontal line. There are two predefined criteria of comfort: at the beginning (no comfort) and finish (maximum comfort). The patient expressed his level of comfort by putting a visible mark on this line. The point marked by the patient was converted to the same decimal number as was the millimeter marked on the line. The decimal number varied from 0 to 10. "Are you comfortable with the mask you're wearing?" was the precise question. The form recording the VAS had detailed instructions written on it to elaborate the method to express the VAS.

The primary outcome noted was the proportion of patients who were intubated. The secondary outcomes were duration of NIV, ICU length of stay, in hospital mortality, $\mathrm{PaO}_{2} / \mathrm{FiO}_{2}$ ratio, and patient comfort and complications.

The results of the study were entered into the SPSS version 23 programmed and checked for discrepancies and outliers. The Shapiro Wilk Test was used to ensure that the data were normal. The mean and standard deviation were used to express quantitative variables with a normal distribution. Proportions or percentages were used to express the categorical variables. The proportions were compared using Chi-square or Fisher's exact test, and the mean among both the intervention groups was comparatively 
analyzed using independent $t$-test. Odd's ratio was used to assess the relationship. The Kaplan-Meier plot was generated using SPSS for survival analysis, and other graphs were created using Microsoft Excel version 16. In a Kaplan-Meier plot, the groups were compared using the Log Rank Test. In our investigation, a $p$ value of less than equal to 0.05 was accepted as statistically significant.

\section{RESULts}

Sixty-four patients were approached for the study between March and May 2021; four patients declined to participate, therefore sixty patients were enrolled in the study and were consequently randomized (Flowchart 1). Thirty patients were randomized to group I, the helmet mask, and 30 to group II, the face mask group.

The two study groups were comparable in terms of demographics, clinical features, comorbidities, baseline ABG, hemodynamic parameters, and Simplified Acute Physiology Score (SAPS II) on admission (Table 1).

The rate of endotracheal intubation rate was significantly more in the face mask group in comparison to the helmet mask group (43.3 vs 10\%, $p=0.004$ ) (Table 2). The Kaplan-Meier plot for

Flowchart 1: Consort flow diagram of the study

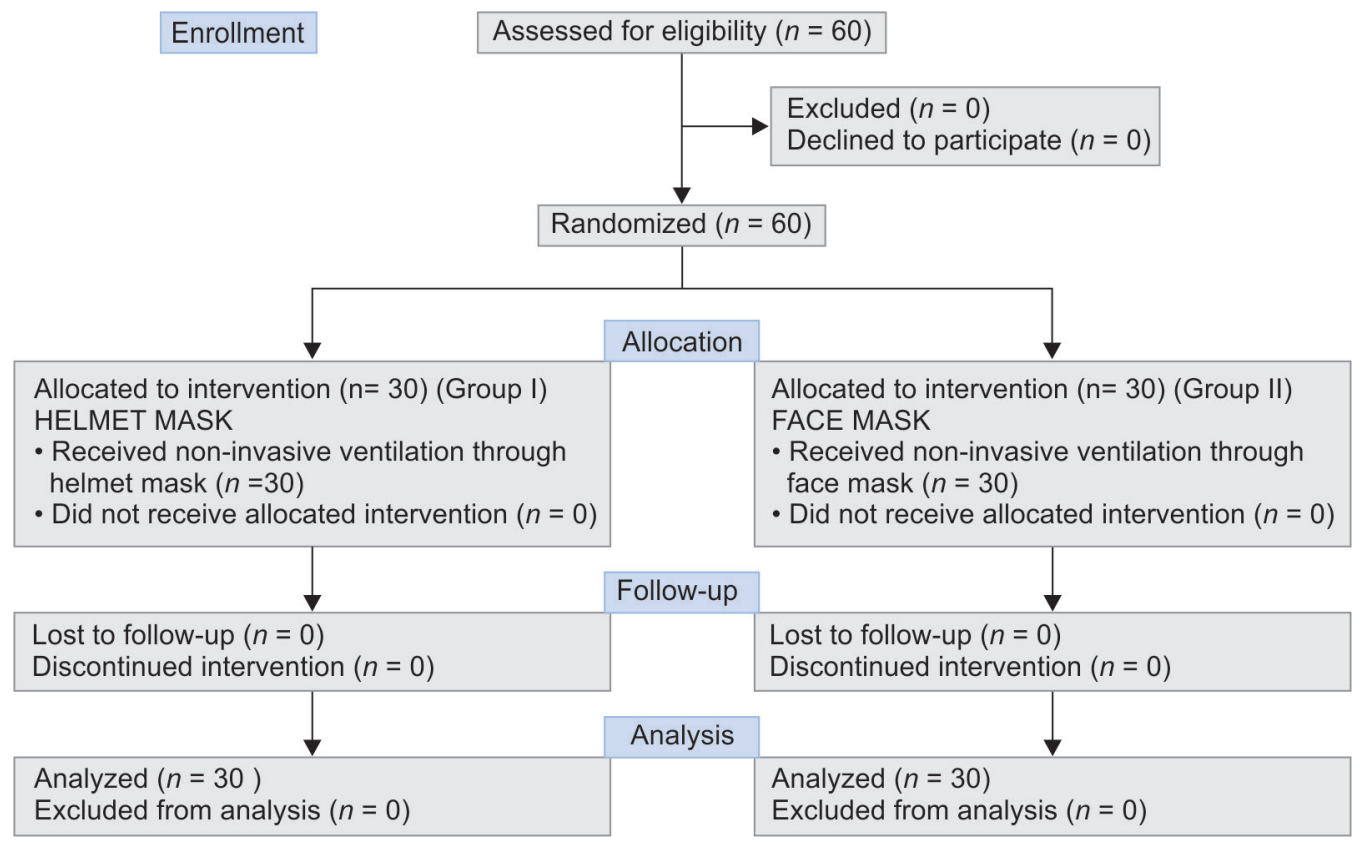

Table 1: Baseline patient characteristics

\begin{tabular}{|c|c|c|c|}
\hline Parameters & $\begin{array}{l}\text { Helmet mask } \\
\quad(N=30)\end{array}$ & $\begin{array}{l}\text { Face mask }^{*} \\
(N=30)\end{array}$ & $p$ value $^{t}$ \\
\hline Gender, male & 18 & 15 & 0.43 \\
\hline Age (years) & $53.23 \pm 9.179$ & $52.56 \pm 9.807$ & 0.787 \\
\hline BMI (median IQR ${ }^{£}$ ) & $24.5(22-27)$ & $23.5(21-26)$ & 1.000 \\
\hline Heart rate & $87.03 \pm 3.567$ & $87.87 \pm 3.371$ & 0.124 \\
\hline Mean arterial blood pressure & $83.63 \pm 1.159$ & $83.33 \pm 1.709$ & 0.429 \\
\hline $\mathrm{PAO}_{2} / \mathrm{FIO}_{2}{ }^{\ddagger}$ & $155.33 \pm 3.836$ & $155.33 \pm 2.975$ & 0.946 \\
\hline Respiratory rate & $32.77 \pm 1.165$ & $32.37 \pm 1.066$ & 0.171 \\
\hline $\mathrm{SpO}_{2}^{\S}$ & $84.47 \pm 1.479$ & $84.6 \pm 1.221$ & 0.355 \\
\hline SAPS $\|^{*}$ & $32 \pm 9$ & $31 \pm 9$ & 0.668 \\
\hline \multicolumn{4}{|l|}{ Comorbidities, $n$ (\%) } \\
\hline Diabetes & $10(33.3)$ & $12(40)$ & 0.592 \\
\hline Hypertension & $12(40)$ & $14(46)$ & 0.602 \\
\hline Coronary artery disease & $3(10)$ & $5(16.6)$ & 0.451 \\
\hline Chronic kidney disease & $2(6.6)$ & $3(10)$ & 0.642 \\
\hline Chronic liver disease & $1(3.3)$ & 0 & 0.493 \\
\hline
\end{tabular}

${ }^{*}$ Values are presented as mean \pm SD. ${ }^{\dagger} p$ value less than 0.05 is considered significant. ${ }^{\ddagger} \mathrm{PaO}_{2} / \mathrm{FiO}_{2}$, ratio of partial pressure of oxygen and fraction of inspiratory oxygen concentration; ${ }^{\S} \mathrm{SpO}_{2}$, saturation of oxygen; ${ }^{\mathrm{f}} \mathrm{IQR}$, interquartile range; ${ }^{\sharp}$ Simplified Acute Physiology Score (SAPS) II calculator scores ranges from 0 to 163, with higher scores reflecting greater disease severity 
Table 2: Primary and secondary outcomes

\begin{tabular}{lcccl}
\hline Parameters & Helmet mask, numbers (\%) & Face mask, numbers (\%) & p value & \multicolumn{1}{c}{ Odds ratio } \\
\hline Endotracheal intubation & $3 / 30(10 \%)$ & $13 / 30(43.3 \%)$ & 0.004 & $1.707-27.752$ \\
In-hospital mortality & $4(13.3 \%)$ & $12(40 \%)$ & 0.020 & $1.23-15.60$ \\
Complications & $3 / 30(10 \%)$ & $13 / 30(43.3 \%)$ & 0.004 & $1.707-27.752$ \\
\hline
\end{tabular}

$p$ value less than 0.05 is considered significant

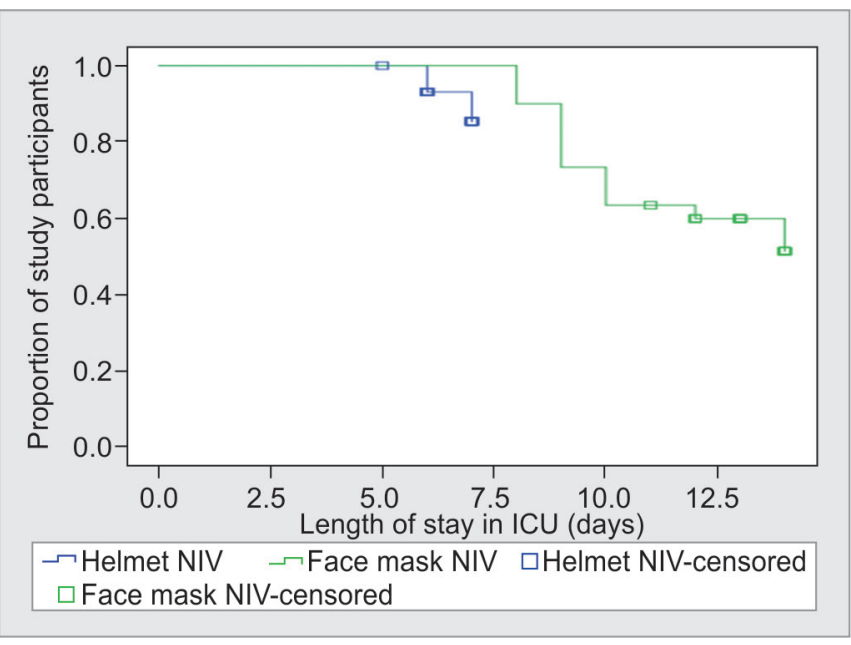

Fig. 1: Survival analysis

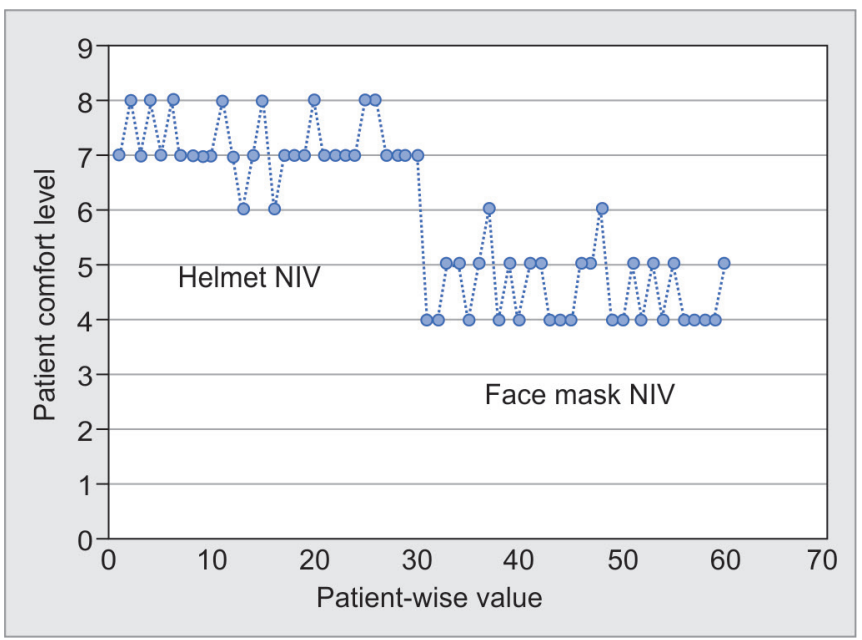

Fig. 3: Graphical representation of patient comfort on both the interfaces

intubation as an event showed significant difference in duration and proportion of participants undergoing intubation in both the study groups of this study (Fig. 1).

During the detailed analysis of the secondary outcomes, the helmet mask group patients had decreased duration of NIV and spent lesser days in ICU as compared to the patients in the face mask group $(4.53 \pm 0.776$ vs $7.60 \pm 1.354, p=0.00$ and $6.37 \pm 0.556$ vs $11.57 \pm 2.161, p=0.00$, respectively).

The use of the helmet mask significantly improved the $\mathrm{PaO}_{2} / \mathrm{FiO}_{2}$ ratio throughout the clinical course of patients as compared to the face mask group ( $263.57 \pm 31.562$ vs $209.33 \pm 20.531, p=0.00$; Fig. 2 ). Respiratory rate in the helmet group $(22.53 \pm 1.697)$ was also significantly lower than in the face maskgroup $(27.37 \pm 1.159)(p=0.00)$.

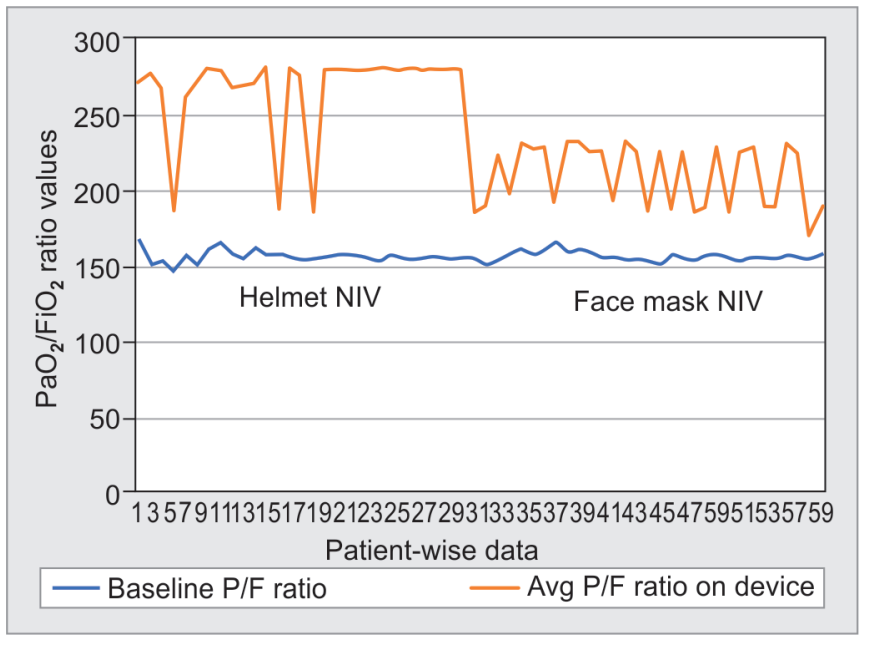

Fig. 2: Graphical representation of $\mathrm{PaO}_{2} / \mathrm{FiO}_{2}$ ratio of both the interfaces

The patient comfort was more with the use of helmet mask than the face mask as detailed by the VAS score $(7.20 \pm 0.551$ vs $4.53 \pm$ $0.629, p=0.00$; (Fig. 3). No significant difference in heart rate and blood pressure was observed between the groups (Table 3).

The in-hospital mortality was significantly less with the use of helmet mask NIV (13.3 vs $40 \%, p=0.020)$. Thirteen patients (43.33\%) in the face mask group developed ulceration over the bridge of the nose and cheeks as compared to three patients (10\%) in the helmet mask group who complained of increased operating noise during helmet use ( $p=0.004$; Table 2).

\section{Discussion}

NIV has been a well-established ventilatory support mode for patients with respiratory failure secondary to chronic obstructive pulmonary disease and acute cardiogenic pulmonary edema, but the evidence supporting its use in hypoxia secondary to ARDS is limited. ${ }^{7}$ Continuous positive airway pressure and NIV are routinely used to treat patients with moderate-to-severe acute respiratory failure secondary to COVID-19. Newer methods for respiratory assistance are being explored and hence an enhanced use and understanding of NIV is worthwhile.

In this single center, randomized open labeled clinical trial, the primary outcome noted was comparison of rate of endotracheal intubation among the two groups. The findings of our research back up our previous supposition. The patients who were given NIV by helmet mask, required lesser intubation, in comparison to the patients who were given NIV by face mask (10 vs $43.3 \%, p=0.004$ ). Our results are in agreement with the previous studies in which non-COVID patients of ARDS managed on helmet mask showed a better outcome with respect to intubation rates and oxygenation. ${ }^{5,8}$

On analysis of secondary outcomes in our study, the patients on helmet masks showed improved oxygenation and lower 
Table 3: Primary and secondary outcomes

\begin{tabular}{lccccc}
\hline Parameters & Helmet mask $^{*}$ & Face mask $^{*}$ & p value $^{\dagger}$ & Mean difference & Confidence interval \\
\hline Duration of NIV & $4.53 \pm .77$ & $7.60 \pm 1.35$ & 0.00 & -3.06 & $-3.63-(-2.49)$ \\
Length of ICU stay & $6.37 \pm 0.55$ & $11.57 \pm 2.16$ & 0.00 & -5.200 & $-6.01-(-4.38)$ \\
$\mathrm{PAO}_{2} / \mathrm{FIO}_{2}{ }^{*}$ & $263.57 \pm 31.56$ & $209.33 \pm 20.53$ & 0.00 & 54.233 & $40.47-67.99$ \\
Respiratory rate & $22.53 \pm 1.69$ & $27.37 \pm 1.15$ & 0.00 & -4.83 & $-5.58-(-4.08)$ \\
Heart rate & $84.17 \pm 1.48$ & $83.93 \pm 1.28$ & 0.35 & 0.23 & $-0.48-0.95$ \\
Mean arterial blood pressure & $82.6 \pm 1.65$ & $83.03 \pm 1.09$ & 0.46 & -0.43 & $-1.15-0.29$ \\
Patient comfort & $7.20 \pm .551$ & $4.53 \pm .62$ & 0.00 & 2.66 & $2.36-2.97$ \\
\hline
\end{tabular}

*Values are presented as mean and standard deviation. ${ }^{\dagger} p$ value less than 0.05 is considered significant; ${ }^{\ddagger} \mathrm{PaO}_{2} / \mathrm{FiO}_{2}$, ratio of partial pressure of oxygen and fraction of inspiratory oxygen

respiratory rate as compared to the patients on face mask $(p=0.00)$. Effective generation of positive-end expiratory pressure by helmet mask as compared to face mask ${ }^{9,10}$ may have led to opening of non-ventilated alveoli at the lung bases, increasing the functional residual capacity and a reduction in the shunt fraction. Studies have also pointed out that the use of helmet mask for NIV reduces the work of breathing by increasing the synchrony between patient and ventilator. The shifting of the tidal volume to a higher compliance point of the pressure-volume curve may be responsible for this better synchronization. ${ }^{11}$ The use of a helmet mask made prone positioning simple, safe, and practicable, which could be another cause for increased oxygenation and shorter NIV duration. ${ }^{12,13}$ In the early phases of the disease, continuous application of NIV is of paramount importance. With the use of helmet mask, the interruptions in NIV during drinking, communication, or clearing of sputum are minimized. This could have contributed to reduction in the total duration of NIV, length of ICU stay, and in-hospital mortality $(p=0.00)$.

Similar results were concluded by a study by Kyeremanteng $\mathrm{K}$ et al., on the comparison of cost analysis between NNV by helmet mask and face mask in ARDS. They reported that the use of the helmet mask interface in ARDS patients reduced the rate of intubation and mortality. The ICU stay and the resulting hospital costs were also decreased. ${ }^{14}$ This is of particular importance in the testing times of pandemic when there is shortage of ICU beds.

The use of helmet mask has been reported to have higher patient comfort and lower complication rate. In our study also, the helmet mask group reported better comfort level than those in the face mask group $(p=0.00)$. The complication rate with the use of helmet mask interface was significantly less as compared with the use of face mask ( 10 vs $43 \%, p=0.004$ ). Previous studies on long-term NIV use with face mask interface have also reported that development of ulcerative lesions on face and nasal bridge is frequently responsible for NIV failure in these patients. ${ }^{15}$

Helmet mask has no bony contact leading to decreased facial lesions as compared to face mask. Another reason for higher patient compliance with helmet mask is that it allows enteral nutrition and hydration. ${ }^{16}$ The use helmet mask allows the humidified expired gases to combine with the dry fresh gas flow. This raises the temperature and humidity of the inspired gases, improving patient comfort without the need for active humidification. ${ }^{17}$ A few patients in the helmet mask group reported increased operating noise as a barrier to helmet use. Use of ear plugs by the patients on helmet mask reduced this discomfort. ${ }^{18}$

Concerns about aerosol dispersion have been a major sticking point for NIV usage in COVID-19 patients. In comparison to HFNC or face mask, helmet masks have been shown to considerably reduce SARS-CoV-2 aerosolization and exposure risk for healthcare staff. The lower aerosol dispersion associated with helmet mask may be attributed to a better fit at the neck and fewer air leaks because it is not affected by the face anatomy of the patient. The use of heat-moisture exchange and high-efficiency particulate air filters attached to both the limbs of the circuit may further limit aerosolization. ${ }^{19}$

A similar study on COVID-19 ARDS patients has not been found to our knowledge, despite a thorough literature search. There are some studies and independent metanalysis evaluating the effectiveness of helmet mask as an interface for providing NIV in patients of ARDS, before the emergence of COVID-19. ${ }^{20-23}$ They advocated for the use of a helmet mask as an effective treatment modality for respiratory failure, claiming that it improved the $\mathrm{PaO}_{2} / \mathrm{FiO}_{2}$ ratio, reduced intubation rates, and reduced in-hospital mortality. However, there were no randomized controlled trials that included COVID-19 participants for this comparison.

As we did an open-label trial, there is still a substantial risk of information bias. To minimize this effect, the variables chosen were objective. The presence of a small sample of patients from a single center could be another limitation. Third, we could not quantify the aerosol dispersion with either of the two methods of NIV due to the unavailability of device, technique, and expertise to measure the aerosol dispersion.

Helmet masks are a treatment alternative that has been utilized in Italy in the past, as well as during the COVID-19 pandemic. ${ }^{24}$ However, there was no consensus in the latest Surviving Sepsis Campaign guidelines on its clinical benefit in COVID-19. ${ }^{25}$ This study attempts to validate the feasibility and advantages of the use of helmet masks as compared to face mask for NIV in patients developing ARDS due to COVID-19.

\section{ConClusion}

COVID-19 pandemic has been a time of unparalleled medical challenges. In this regard, we suggest that an alternative respiratory support device like a helmet mask could be a feasible interface for the delivery of NIV. It results in a lower rate of endotracheal intubation, better oxygenation with greater patient comfort, and shorter ICU stay as compared to the face mask used for NIV. To generate more robust evidence, large and multi-center randomized controlled trials are required.

\section{ORCID}

Anupriya Saxena $\odot$ https://orcid.org/0000-0002-6203-3962

Nazia Nazir @ ittps://orcid.org/0000-0001-6950-2111 
Ruchi Pandey (1) https://orcid.org/0000-0002-4653-6738

Savita Gupta @ https://orcid.org/0000-0002-3104-7368

\section{References}

1. Grasselli G, Tonetti T, Protti A, Langer T, Girardis M, Bellani G, et al. Pathophysiology of COVID-19-associated acute respiratory distress syndrome: a multicentre prospective observational study. Lancet Respir Med 2020;8(12):1201-1208. DOI: 10.1016/S2213-2600(20)30370-2.

2. Bertaina $M$, Nuñez-Gil IJ, Franchin L, et al. Non-invasive ventilation for SARS-CoV-2 acute respiratory failure: a subanalysis from the HOPE COVID-19 registry. Emerg Med J 2021;38:359-365. DOI: 10.1136/ emermed-2020-210411.

3. Amirfarzan H, Cereda M, Gaulton TG, Leissner KB, Cortegiani A, Schumann R, et al. Use of Helmet CPAP in COVID-19. A practical review. PULMOE-1596. Available from: https://doi.org/10.1016/ j.pulmoe.2021.01.008. DOI: 10.1016/j.pulmoe.2021.01.008.

4. Ing RJ, Bills C, Merritt G, Ragusa R, Bremmer RM, Bellia F. Role of helmetdelivered noninvasive pressure support ventilation in COVID-19 patients. J Cardiothorac Vasc Anesth 2020;34:2575-2579. DOI: 10.1053/j.jvca.2020.04.060.

5. Patel BK, Wolfe KS, Pohlman AS, Hall JB, Kress JP, et al. Effect of noninvasive ventilation delivered by helmet vs face mask on the rate of endotracheal intubation in patients with acute respiratory distress syndrome. Journal of the American Medical Association 2016;315(22):2435-2441. DOI: 10.1001/jama.2016.6338.

6. Mc Cormack HM, Horne DJ, Sheather S. Clinical applications of visual analogue scales: a critical review. Psychol Med 1988;18:1007-1019. DOI: $10.1017 /$ s0033291700009934.

7. McNeill G, Glossop AJ. Clinical applications of non-invasive ventilation in critical care. Continu Educ Anaesth Crit Care Pain 2012;12(1):33-37. Available from: https://doi.org/10.1093/bjaceaccp/mkr047.

8. Luo Y, Luo Y, Li Y, Zhou L, Zhu Z, Chen Y, et al. Helmet CPAP versus oxygen therapy in hypoxemic acute respiratory failure: a metaanalysis of randomized controlled trials. Yonsei Med J 2016;57(4): 936-941. DOI: 10.1007/s00134-014-3325-5.

9. Patroniti N, Foti G, Manfio A, Coppo A, Bellani G, Pesenti A, et al. Head helmet versus face mask for non-invasive continuous positive airway pressure: a physiological study. Intens Care Med 2003;29:1680-1687. DOI: 10.1007/s00134-003-1931-8.

10. Grieco DL, Menga LS, Cesarano M, et al. Effect of helmet noninvasive ventilation vs high-flow nasal oxygen on days free of respiratory support in patients with COVID-19 and moderate to severe hypoxemic respiratory failure: the HENIVOT Randomized Clinical Trial. Journal of the American Medical Association 2021;325(17):1731-1743. DOI: 10.1001/jama.2021.4682.

11. Rodriguez AME, Papadakos PJ, Carron M, Cosentini R, Chiumello D. Clinical review: helmet and non-invasive mechanical ventilation in critically ill patients. Critic Care 2013;17:223. DOI: 10.1186/cc11875.

12. Raoof S, Master FCCP, Nava S, Carpati C, Hill NC. High-flow, noninvasive ventilation and awake (nonintubation) proning in patients with coronavirus disease 2019 with respiratory failure. Chest 2020;158(5):1992-2002. Available from: https://doi.org/10.1016/ j.chest.2020.07.013.
13. Retucci M, Aliberti S, Ceruti C, Santambrogio M, Tammaro S, Cuccarini F, et al. Prone and lateral positioning in spontaneously breathing patients with COVID-19 pneumonia undergoing non-invasive helmet CPAP treatment. Chest 2020;158(6):2431-2435. Available from: https:// doi.org/10.1016/j.chest.2020.07.006.

14. Kyeremanteng K, Gagnon LP, Robidoux R, Thavorn K, Chaudhuri D, Kobewka D, et al. Cost analysis of noninvasive helmet ventilation compared with use of noninvasive face mask in ARDS. Canad Resp J 2018; Article ID: 6518572. paghttps://doi.org/10.1155/ 2018/6518572.

15. Liu Q, Shan M, Zhu HO, et al. Noninvasive ventilation with a helmet in patients with acute respiratory failure caused by chest trauma: a randomized controlled trial. Sci Report 2020;10:21489. DOI: 10.1186/ s13054-016-1449-4.

16. Kogo M, Nagata K. Enteral nutrition during noninvasive ventilation: we should go deeper in the investigation. Resp Care 2017;62(8). DOI: 10.4187/respcare.05684.

17. Chiumello D, Chierichetti M, Tallarini F, Cozzi P, Cressoni M, Polli F, et al. Effect of a heated humidifier during continuous positive airway pressure delivered by a helmet. Crit Care 2008;12(2):55. DOI: 10.1186/ cc6875.

18. Cavaliere F, Conti G, Costa R, Proietti R, Sciuto A, Masieri S, et al. Noise exposure during noninvasive ventilation with a helmet, a nasal mask, and a facial mask. Intens Care Med 2004;30:1755-1760. DOI: 10.1007/ s00134-004-2347-9.

19. Hui DS, Chow BK, Lo T, Ng SS, Ko FW, Gin T, et al. Exhaled air dispersion during noninvasive ventilation via helmets and a total face mask. Chest 2015; 147(5):1336-1343. DOI: 10.1378/chest.14-1934.

20. Liu Q, Gao Y, Chen R, Cheng Z. Noninvasive ventila-tion with helmet versus control strategy in patients with acute respiratory failure: a systematic review and meta-analysis of controlled studies. Crit Care 2016;20(1):265. DOI: 10.1186/s13054-016-1449-4.

21. Brambilla AM, Aliberti S, Prina E, Nicoli F, Forno MD, Nava S, et al. Helmet CPAP vs oxygen therapy in severe hypoxemic respiratory failure due to pneumonia. Intens Care Med 2014;40:942-949. DOI: 10.1007/s00134-014-3325-5.

22. Cosentini R, Brambilla AM, Aliberti S, Bignamini A, Nava S, Maffei A et al. Helmet continuous positive airway pressure vs oxygen therapy to improve oxygenation in community-acquired pneumonia: a randomized, controlled trial. Chest 2010;138:114-120. DOI: 10.1378/ chest.09-2290.

23. Chiumello D, Pelosi P, Carlesso E, Severgnini P, Aspesi M, Gamberoni C, et al. Noninvasive positive pressure ventilation delivered by helmet vs standard face mask. Intens Care Med 2003;29:1671-1679. DOI: 10.1007/s00134-003-1825-9.

24. Aliberti S, Radovanovic D, Billi F, Sotgiu G, Costanzo M, Pilocane T, et al. Helmet CPAP treatment in patients with COVID-19 pneumonia: a multicenter, cohort study. Eur Resp J 2020. DOI: 10.1183/13993003.01935-2020.

25. Salim Rezaie. "Surviving Sepsis Campaign Guidelines on the Management of Critically III Adults with COVID-19", REBEL EM blog, March 22, 2020. Available at: https://rebelem.com/surviving-sepsiscampaign-guidelines-on-the-management-of-critically-ill-adultswith-covid-19/. 